\title{
The German Breast Group - Healing Through Innovation, Competence and Partnership
}

\author{
Mattea Linder, ${ }^{1}$ Sibylle Loibl² and Gunter von Minckwitz ${ }^{3}$ \\ 1. Medical Research Fellow; 2. Department Leader, Medicine and Research; 3. Managing Director, German Breast Group Research Institute
}

DOI: 10.17925/EOH.2009.05.1.41

\section{Abstract}

The German Breast Group (GBG) is the leading breast cancer study group in Germany, conducting clinical trials in the adjuvant, neoadjuvant, preventative and palliative settings. One focus of the GBG is neoadjuvant studies, which have a long and successful history in Germany. The aim of neoadjuvant studies is to improve operability and to gain more information about the tumour itself in terms of the chemotherapy (in vivo) as well as regarding the biology in general. However, preventative, palliative and adjuvant studies are also important components of the GBG. This article is an overview of the structure and working practice of the GBG and gives a sample of trials currently being led by the GBG.
\end{abstract}

\section{Keywords}

Breast carcinoma, chemotherapy, German Breast Group, Germany, study group, targeted therapy, translational research

Disclosure: Mattea Linder has no conflicts of interests to declare. Sibylle Loibl and Gunter von Minckwitz have received speakers' honoraria from sanofi-aventis, Roche and Amgen. Gunter von Minckwitz has received research funding from all manufacturers whose products were involved in the presented studies. Received: 26 June 2009 Accepted: 31 July 2009

Correspondence: Mattea Linder, Ärztin med. Beratung, GBG Forschungs GmbH, Schleussnerstrasse 42, 63263 Neu-Isenburg, Germany.

E: mattea.linder@germanbreastgroup.de

The German Breast Group (GBG) is a leading study group covering the complete field of clinical breast cancer research in Europe. It was created out of the German Adjuvant Breast Group (GABG), established in 1981. Since 2003 the GBG has been restructured and since the late 1990s not only adjuvant but also neoadjuvant breast cancer trials, as well as prevention and palliation trials, have been launched. The GBG provides comprehensive management of clinical trials in all major therapeutic categories: prevention, neoadjuvant, adjuvant and palliative. The vision of the GBG is best described as healing by innovation, competence and partnership.

The studies are designed and surveyed by the members of the subboards (neoadjuvant, adjuvant/preventative, palliative and the most recent, surgical). The sub-board members are physicians and experts in the field of breast cancer, contributing their knowledge to innovation and trendsetting in study designs. Regular safety controls are supervised by the Independent Data Monitoring Committee (IDMC). Staff meetings take place regularly to update all participants, to discuss the progress of the study and to be able to react quickly in case of safety concerns or in case of new findings that need to be implemented in the study design.

A network of more than 650 national and international centres participates in these trials. In 2008 more than 4,250 patients were enrolled into GBG studies, which is about $8.5 \%$ of all newly diagnosed breast cancer patients in Germany. Overall, about $11 \%$ of all primary breast cancer patients receive their (neo)adjuvant therapy within a clinical trial. Figure 1 shows the overall recruitment in the last 15 years. Since 1981, about 60 trials have been run and about 22,000 patients have been enrolled and treated in trials by the GBG (and the preceding GABG). At the moment, there are 21 trials open for recruitment (three in the neoadjuvant, 11 in the adjuvant, five in the palliative and two in the preventative setting), and over 40 clinical trials are currently being managed by the GBG. The Arbeitsgemeinschaft Gynäkologische Onkologie (AGO)Breast Study Group is joining many of the GBG's trials and using its trial infrastructure.

\section{Neoadjuvant Studies}

The focus of the GBG is the neoadjuvant field, which has a long history in Germany. Since the first studies in the mid-1990s this has been a story of success. The reason neoadjuvant studies in breast cancer are particularly popular and successful probably lies in the way breast cancer is treated in Germany. Diagnostic, systemic and surgical treatment lies in general in the hands of the gynaecologist, who co-operates in a multidisciplinary team. Therefore, there is a single point of contact for the patients, which reduces time. Innovative strategies, especially the neoadjuvant concept, could be implemented into daily practice via a tight clinical trial network. The advantages of such treatment designs are increased rates of breastconserving therapy, in vivo testing of chemosensitivity, and therefore prediction of the patient's prognosis, and gaining more information about the tumour biology with translational research projects.

The GEPARDO study, the first neoadjuvant GBG trial, was published in 2001 in the Journal of Clinical Oncology. ${ }^{1}$ The study was designed to investigate the possibility of the combination of doxorubicin and docetaxel \pm tamoxifen $(A D O C \pm T a m)$ regardless of hormone receptor status, assuming that tamoxifen would overcome resistance to chemosensitivity. This regimen was further investigated in a series of 
Figure 1: Overall Recruitment Since 1994

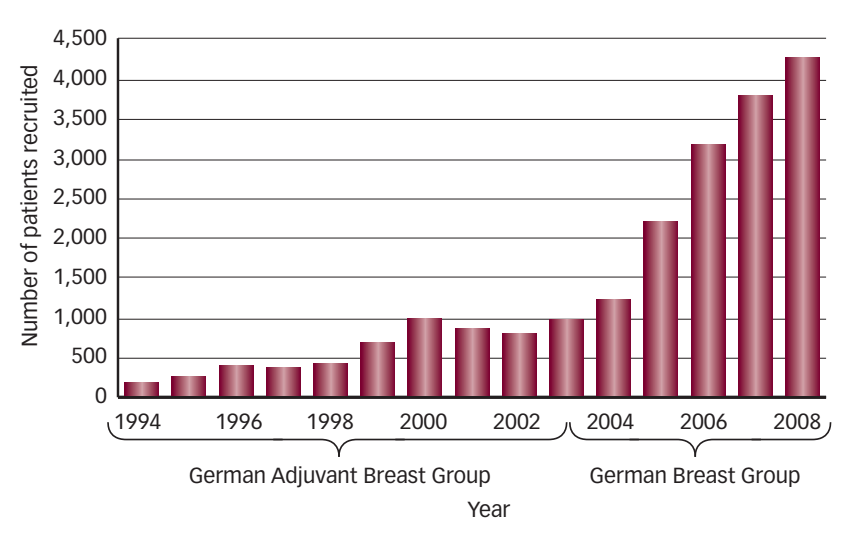

neoadjuvant trials. The first trial (GEPARDO study) demonstrated that tamoxifen had neither a negative nor a positive effect on the pathological complete response (pCR). The GEPARDUO trial had a similar design to the NSABP-B27 study in the US, with patients given doxorubicin plus docetaxel $\mathrm{q} 2 \mathrm{~W}+$ granulocyte colony-stimulating factors (G-CSF) or doxorubicin plus cyclophosphamide followed by docetaxel q3w. This trial showed that sequential neoadjuvant doxorubicin/cyclophosphamide followed by docetaxel (AC-Doc) is more effective at inducing $\mathrm{PCR}$ than dose-dense $A D O C$ as preoperative treatment for patients with operable breast cancer. ${ }^{2}$ The PCR rate could be increased to $14 \%$ with the longer treatment in this setting. As studies have shown a significant correlation between the PCR and disease-free survival (DFS), it is fundamental for patients to further improve the tumour response to chemotherapy. ${ }^{3.4}$

The subsequent GeparTrio ${ }^{5}$ study evaluated the neoadjuvant approach as an in vivo chemosensitivity test and divided the treatment groups according to clinical response (mid-course response) after two cycles of docetaxel, doxorubicin and cyclophosphamide (TAC). The pilot study focused on the feasibility and validity of the study, whereas the main trial in phase III, which included 2,072 patients, evaluated a more intense regimen in the group of responders (6xTAC versus 8xTAC) as well as non-responders (2XTAC followed by $4 x$ vinorelbine and capecitabine [NX] versus $6 x$ TAC). Overall, no advantage could be detected either in the responders group or in the non-responders group for the more intense regimen, but subgroup analyses in the GeparTrio study that were recently presented at the American Society of Clinical Oncology (ASCO) meeting in $2009^{\circ}$ showed that age $<40$ years, poor grading, a non-lobular histology and triple-negative tumours were independent predictors of a pCR.

The GeparQuattro trial was started in August 2005, before the GeparTrio trial was finally analysed; therefore, the sequential regimen AC-Doc was further developed, investigating the additional benefit of capecitabine either in sequence or in combination with docetaxel in 1,510 patients. Her2-positive patients received, in a non-randomised fashion, trastuzumab from the very beginning of the chemotherapy. The safety analysis demonstrated that this approach was feasible without increasing the cardiac side effects, but with an almost doubled pathological complete response rate.?

Therefore, the GeparQuinto trial (goal $n=2,500$ patients), which is currently recruiting, is evaluating in a head-to-head comparison whether trastuzumab or lapatinib as anti-Her2 agent in the neoadjuvant setting should be favoured. Her2-negative patients are randomised to 4 XEC followed by four $x$ docetaxel with or without bevacizumab. After completing the first four cycles of $\mathrm{EC}$, a response evaluation by ultrasound will separate the responding from the non-responding group. The latter will stop bevacizumab and be randomised to paclitaxel weekly with or without the mTor inhibitor RAD001 (everolimus). The responder group will continue according to the initial randomisation with or without bevacizumab and continue chemotherapy with docetaxel (ECDOC \pm bevacizumab). The rate of pathological complete response, defined as no invasive tumour in the breast and axillary lymph nodes (ypTO/Tis; ypNO), is the primary end-point in this trial.

In 2002 the AGO started its first neoadjuvant trial (AGO 1 study), elaborating the dose-dense approach in the neoadjuvant setting. It was demonstrated that the dose-dense treatment (ddE-ddP) followed postoperatively by cyclophosphamide/methotrexate/5-fluorouracil (CMF) is superior not only in terms of improved PCR rates but also in terms of improved long-term outcome. ${ }^{8}$ The PReoperative Epirubicin Paclitaxel ARanEsp (PREPARE) trial, which started thereafter, also demonstrated a superior PCR rate for the dose-dense approach (ddE-ddP-CMF) compared with standard EC-P. In parallel, the Taxol Epirubicin Cyclophosphamid Herceptin NeOadjuvant (TECHNO) trial evaluated the addition of trastuzumab to standard EC-P, starting with trastuzumab after completing the treatment including antracycline. The GBG entered into the conduct of these two trials at a later time-point.

All eight trials (five by the GBG and three by the AGO) have been incorporated into one database. The first preliminary results of more than 6,000 patients treated with an antracycline- and taxanecontaining chemotherapy in the neoadjuvant setting were presented at last year's San Antonio Breast Cancer Symposium (SABCS). ${ }^{9}$ The two groups have joined forces: the GeparQuinto and the GeparQuattro study are both intergroup trials of the GBG and the AGO, enabling better recruitment and a more sophisticated translational programme. More details are provided in Table 1.

Another neoadjuvant study of the GBG is the SOFIA study, with a primary focus on translational outcome. The study investigates the small molecule sorafenib - which acts via inhibition of the Raf/Ras kinases as well as neoangiogenesis - in combination with EC-P weekly. ${ }^{10}$ Sorafenib may be a promising agent to treat patients with locally advanced, previously untreated primary breast cancer, as it has demonstrated single-agent activity in multiple phase I/II studies and was tolerated and inhibited tumour growth in patients with refractory solid tumours, including breast cancer. In several fixed combinations of chemotherapy, sorafenib-treated patients showed a partial response, with most of the patients reaching stable disease. Therefore, the SOFIA study was designed, in which patients received sorafenib $800 \mathrm{mg}$ per day concomitantly with 4 XEC-P weekly. After the first 12 patients, the protocol needed to be amended due to a high rate of skin toxicity. A dose escalation for sorafenib starting with $200 \mathrm{mg}$ per day and increasing stepwise to $800 \mathrm{mg}$ if tolerated was included. This single-arm phase II study with the primary end-point of the amount of PCR at the time of operation is an example of the close relationship between translational research projects and clinical benefit.

\section{Adjuvant Studies}

To further improve adjuvant treatment of early node-positive breast cancer is a permanent goal. A modern and biologically highly 
Table 1: Overview of the Neoadjuvant trials of the German Breast Group Since 1998

\begin{tabular}{|c|c|c|c|c|}
\hline Trial & Publication & $n=6,708$ & $\begin{array}{l}\text { Recruitment } \\
\text { Period }\end{array}$ & Therapy \\
\hline GEPARDO & von Minckwitz et al., JCO, 2001 & 248 & 04/98-06/99 & $\mathrm{ADOC} \pm \mathrm{Tam}$ \\
\hline GEPARDUO & von Minckwitz et al., JCO, 2001 & 907 & 06/99-09/01 & ADOC q2w vs AC-Doc q3w ( \pm Tam) \\
\hline GeparTrio Pilot & von Minckwitz et al., Annals Oncol, 2005 & 285 & 09/01-09/02 & $2 \times$ TAC responder: 4 x TAC; non-responder: 4 x TAC vs 4 x NX \\
\hline GeparTrio & von Minckwitz et al., Annals Oncol, 2005 & 2,072 & 07/02-07/05 & $2 \times$ TAC responder: 4 x TAC vs 6 X TAC; non-responder: 4 x TAC vs 4 NX \\
\hline GeparQuattro & von Minckwitz et al., Untch et al., EBCC, 2008 & 1,495 & 08/05-12/06 & EC-Doc vs EC-DOCX vs EC - Doc-X if Her2- pos: + Tras \\
\hline AGO 1 & Untch et al., JCO, in press & 668 & 06/98-04/02 & EP q3W vs ddE-ddP followed post-operatively by CMF \\
\hline PREPARE & Untch et al., ASCO, 2008 & 733 & 06/02-02/05 & EC-P vs Edd-Pdd-CMF \\
\hline TECHNO & Untch et al., SABCS, 2005 & 230 & 06/02-02/05 & EC-P (+ Tras, if Her2 pos) continuation of Tras post-operation \\
\hline
\end{tabular}

$A D O C=$ doxorubicin and docetaxel; Tam = tamoxifen; $A C-D O C=$ neoadjuvant doxorubicin/cyclophosphamide followed by docetaxel; TAC = docetaxel, doxorubicin and cyclophosphamide; $N X=$ vinorelbine and capecitabine; $E C-D O C=E 90 C_{600}$ docetaxel; Tras = trastuzumab; $E C-P=$ epirubicin/cyclophosphamide followed by paclitaxel; EP = epirubicin $/ p a c l i t a x e l ; d d E-d d P=$ dosedense treatment; $\mathrm{CMF}=$ cyclophosphamide/methotrexate/5-fluorouracil.

Table 2: Overview of Biologicals Currently Used in German Breast Group Trials

\begin{tabular}{lll} 
Substance & Trial & Target Point \\
Trastuzumab & Geparquinto, Neo-Altto & Antibody: anti-Her2-neu \\
\hline Lapatinib & Geparquinto, Neo-Altto & TKl: anti-Her-1 and 2 \\
\hline Pertuzumab & Study in preparation & Antibody: HER dimerisation inhibitor (HER1, HER2, HER3 and HER4) \\
\hline Rad001 & Geparquinto & mTOR inhibitor \\
\hline Sorafenib & SOFIA & TKl: anti-RAF/Ras, VEGF \\
\hline Denusomab & Study in preparation & Antibody: RANK ligand inhibitor \\
\hline Bevacizumab & Geparquinto & Antibody: anti-VEGF \\
\hline Nab-Paclitaxel & ICE II & Inhibitor of mitosis \\
\hline
\end{tabular}

TKI = tyrosinkinase inhibitor; VEGF = vascular endothelial growth factor; mTOR = mammalian target of rapamycin.

interesting concept to achieve this is the dose-dense, doseintensified strategy with chemotherapy delivered every second week instead of every third week. The delivery of chemotherapy every third or fourth week has been guided by its side effects rather than tumour biological considerations. The use of the cytokine G-CSF has facilitated the delivery of chemotherapy every second week and, surprisingly, the data from these studies (CALGB, Möbus et al.) did not demonstrate increased side effects from this procedure. More frequent delivery of chemotherapy reduces the non-exposure interval in which re-growth and neo-angiogenesis may occur, and thus a better outcome is expected.

The German Adjuvant Intergroup Node-positive (GAIN) study was the first GBG study investigating this approach. It was a challenging and very successful GBG study, in which more than 3,000 patients were included, and has just finished recruitment. It was a multicentre phase III trial to compare ETC versus EC-TX with or without ibandronate in patients with node-positive primary breast cancer. The primary end-point was DFS. A few amendments had to be performed during the course of the study: implementation of prophylactic ciprofloxacin 500mg by mouth during EC and C, dose adjustments for cyclophosphamide (from 2,500 to 2,000mg/m²), administration of pegfilgrastim on day two versus day four and, finally, the integration of a dose adaptation to erythropoesisstimulating factors in correspondence with the haemoglobin level. The first safety analysis of all randomised patients demonstrated that haematological toxicities occurred more commonly with ETC; both regimens seemed feasible if supportive treatment was subsequently given according to protocol, and study discontinuation was more frequent in EC-TX. First efficacy data are expected in 2010.

The PANTHER study is further investigating the dose-dense approach. It is a randomised phase III trial comparing bi-weekly a tailored epirubicin-cyclophosphamide followed bi-weekly by tailored docetaxel (dtEC $\rightarrow \mathrm{dtT}$, arm A) versus three-weekly FEC followed by three-weekly docetaxel (FEC $\rightarrow \mathrm{T}$, arm B) in lymph-nodepositive or high-risk node-negative breast cancer patients. The primary objective is recurrence-free survival (RFS) in a population of 1,520 patients. The study is led by the Scandinavian Breast Cancer Group (SBG) and conducted in collaboration with the Austrian Breast Cancer Study Group (ABCSG).

The GBG has also been undertaking adjuvant research in elderly patients. As the elderly patient is often under-represented in clinical trials but represents a great proportion of affected patients, more data need to be collected regarding safety and efficacy in this population. In the ICE I study, capecitabine \pm ibandronate in the elderly was under investigation. It was shown to be very well tolerated and seemed feasible in this population. The study has just been closed and the first efficacy data are eagerly awaited in 2010. In a meta-analysis the effect of taxanes on elderly patients was evaluated. The study was a joint project together with the AGO, the Westdeutsche Studiengruppe (WSG) and the Nordostdeutsche Gesellschaft für Gynäkologische Onkologie (NOGGO). The ICE ॥ study, which has just begun recruiting, investigates the combination of nab-paclitaxel with capecitabine versus EC or CMF in patients with high-risk primary breast cancer in the elderly either according to a clinicopathological algorithm or by measuring UPA/PAI 1.

\section{Palliative Studies}

In the future the GBG wants to further distinguish between high- and low-risk patients with metastasised breast cancer in order to treat patients according to their disease, thus avoiding overtreatment in a situation where maintaining the patient's quality of life is essential. Therefore, studies were designed taking this into account. 
The RADAR study offers a promising approach for low-risk patients with bone metastasis. RAD001 (everolimus) has long been used for immunosuppressive indications (allocraft reaction) and has been newly discovered as a treatment for patients with advanced or metastasised breast cancer, as it interferes in the regulation of a wide range of growth-related cellular functions, including transcription, translation, membrane protein degradation and reorganisation of the actin cytoskeleton. The RADAR study is a prospective, multicentre, placebo-controlled, randomised discontinuation phase II study. After a run-in period of eight weeks with RAD001 daily, tumour response is evaluated. In case of a complete or partial response, RAD001 intake continues. In case of stable disease with no change, patients will be randomised again to either continue with RAD001 or change to a placebo in a 1:1 ratio. Patients with progressive disease will discontinue the medication and be taken off the study. The primary objective is the time to progression. Another study for patients in the low-risk situation is the LEA study. This trial was designed for hormone-receptor-positive breast cancer patients with any kind of metastasis. In such a situation the German guidelines recommend an antihormonal therapy. Antihormonal therapy in combination with bevacizumab, a vascular endothelial growth factor (VEGF) antibody, is under investigation; a better outcome is expected compared with hormonal therapy alone as first-line treatment for post-menopausal women with metastasised, Her2-negative breast cancer. The trial is being conducted in collaboration with the Spanish group GEICAM.

The TaBeA study is one example for women in the high-risk metastasised situation, and aims to establish a three-drug regimen including two cytotoxic agents and one molecularly targeted drug. It is a randomised phase III study to determine the efficacy of a taxane and bevacizumab with or without capecitabine $1,800 \mathrm{mg} / \mathrm{m}^{2}$ as firstline chemotherapy. As the primary objective is PFS, taxanes are given in combination with bevacizumab until progression or unacceptable toxicity occurs.

Taxanes and capecitabine are all highly active single agents for the treatment of breast cancer and show an acceptable toxicity profile. The addition of the VEGF inhibitor bevacizumab to these cytotoxic agents showed improved response rates for the combination with capecitabine and improved PFS rates for the combination with paclitaxel as well as docetaxel. A combination of all three drugs is therefore considered to be a promising regimen in these high-risk situations of metastatic breast cancer. This trial is again being conducted together with the AGO.

\section{Translational Research}

The GBG has also focused on translational research as a prerequisite for all trials. Neoadjuvant studies are optimal for the incorporation of translational research because collected tumour material from the untreated tumour as well as the treated tumour can be investigated and help to further develop individualised therapy. Since the very first neoadjuvant study, tumour material has been collected, which has enabled us to participate in national as well as international translational research collaborations.
The future will focus even more on projects with a strong translational backbone as this is a fundamental tool for finding answers not only to the biology of breast cancer but also to the treatment of the still life-threatening diagnosis of breast cancer. Therefore, the GBG will focus on further neoadjuvant projects including translational research, and is very hopeful of greatly improving and continuing to standardise breast cancer treatment in Germany. In order to realise this vision, a permanent translational research board has been established to develop new ideas for translational research projects and to evaluate project proposals submitted by third parties.

In 2008 the GBG started two large independently financed national and international scientific collaborations/projects, both in cooperation with Charité University Hospital/Institute of Pathology. First, there is the Neo-Predict project in co-operation with Siemens and financially sponsored by the German Federal Ministry of Education and Research (BMBF), prospectively evaluating molecular biomarker sets in paraffin-embedded tissue for the prediction of response to neoadjuvant chemotherapy in breast cancer. Second, there is the METAcancer project, with the aim of identifying and validating new breast cancer biomarkers based on integrated metabolomics. It is based on further collaborations from Finland, US, UK, Poland and Slovakia and financially sponsored by the EU Framework.

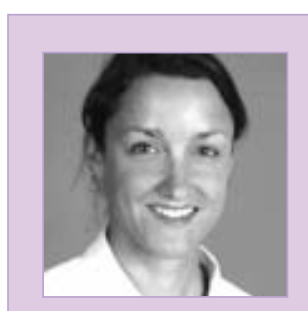

Mattea Linder is a Medical Research Fellow at the German Breast Group (GBG) Research Institute. Her particular interest is male breast cancer and breast cancer in elderly patients. She received her medical education at the Charite University of Berlin and in 2007 finished her dissertation at the same university. She started her fellowship at the Helios Klinikum Berlin Buch in the Department of Gynecology under the supervision of Professor Michael Untch. As part of her education and to deepen her scientific knowledge she became a medical research fellow in the GBG.

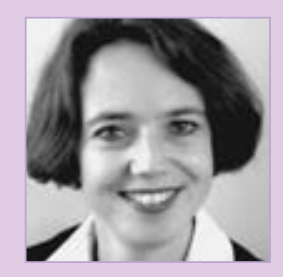

Sibylle Loibl leads the Department of Medicine and Research at the German Breast Group (GBG) Research Institute. She is also an Assistant Professor at the University of Frankfurt and has a once-weekly clinic. Her scientific focus is on breast cancer, particularly breast cancer in pregnancy. In co-operation with Professor Gunter von Minckwitz, Professor Loibl has participated in and led a large number of national and international clinical trials and has contributed to the improvement of the infrastructure of breast cancer trials all over Germany.

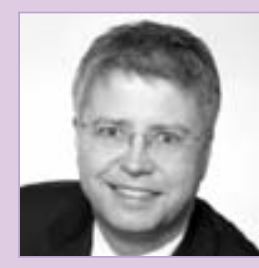

Gunter von Minckwitz is Managing Director of the German Breast Group (GBG) Research Institute. He is also Alternate Director of Senologic Oncology at the Breast Centre in Düsseldorf and a Professor at the University of Frankfurt. Professor von Minckwitz's areas of research include systemic treatment in all stages of the disease (neoadjuvant, adjuvant, metastatic treatment and chemoprevention), and he has participated in and led a large number of national and international clinical trials. He has improved the infrastructure for breast cancer trials all over Germany, and founded and led the development of the national treatment guideline for breast cancer of the Arbeitsgemeinschaft Gynäkologische Onkologie (AGO). The GBG is Germany's largest co-operative group, with approximately 530 centres, 1,100 collaborators and an annual recruitment of about 4,250 breast cancer patients into prospective clinical trials.
1. Von Minckwitz G, et al., J Clin Oncol, 2001;19(15):3506-15.

2. Von Minckwitz G, et al., J Clin Oncol, 2005;23(12):2676-85.

3. Bear HD, et al., J Clin Oncol, 2006;24:2019-27.

4. Smith IC, et al., J Clin Oncol, 2002;20:1456-66.
5. Von Minckwitz G, et al., I Natl Cancer Inst, 2008:100(8):542-51.

6. Huober J, et al., J Clin Oncol, 2009;27:15s, abstract 524

7. Untch M, et al.,Eur J Cancer, 2008;6(7):47.
8. Untch M, et al. Proc Am Soc Clin Oncol, 2002;21:34a.

9. Von Minckwitz G, et al., Abstract Number 79, SABCS 2008.

10. Wilhelm SM, et al., Cancer Res, 2004;64(19): 7099-7109. 